\title{
Hierarchical theory of quantum dissipation: Partial fraction decomposition scheme
}

\author{
Jian Xu and Rui-Xue Xu* \\ Hefei National Laboratory for Physical Science at Microscale, \\ University of Science and Technology of China, Hefei, Anhui 230026, China \\ Meng Luo and YiJing Yan† \\ Department of Chemistry, Hong Kong University of Science and Technology, Kowloon, Hong Kong SAR, China
}

(Dated: December 5, 2018)

\begin{abstract}
We propose a partial fraction decomposition scheme to the construction of hierarchical equations of motion theory for bosonic quantum dissipation systems. The expansion of Bose-Einstein function in this scheme shows similar properties as it applies for Fermi function. The performance of the resulting quantum dissipation theory is exemplified with spin-boson systems. In all cases we have tested the new theory performs much better, about an order of magnitude faster, than the best available conventional theory based on Matsubara spectral decomposition scheme.
\end{abstract}

\section{INTRODUCTION}

It is well established now that path integral influence functional formalism [1, 2, 3] of quantum dissipation theory (QDT) can be reformulated in terms of hierarchical equations of motion (HEOM) [4, 5, 6, 7, 8, 9, 10, 11, 12]. Compared to its dynamics equivalent path integral influence functional formalism, the HEOM has the advantage in both numerical efficiency and applications to various systems. Moreover, the initial system-bath coupling that is not contained in the original path integral formalism can now be accounted for via proper initial conditions to HEOM [11, 12]. As an exact formalism, HEOM is also nonperturbative. It treats the combined effects of system anharmonicity, system-bath coupling strength, and multiple memory time scales on the reduced system dynamics.

The specific HEOM-QDT construction depends however on the way of decomposing bath correlation function into its memory components. Different decomposition schemes are mathematically equivalent, but have different numerical performance. To illustrate this issue, we consider only the single dissipation mode case, in which the system-bath coupling Hamiltonian assumes $H_{\mathrm{SB}}=-Q F_{\mathrm{B}}$. The system operator $Q$ here is also called the dissipation mode, through which the generalized Langevin force $F_{\mathrm{B}}(t) \equiv e^{i h_{\mathrm{B}} t / \hbar} F_{\mathrm{B}} e^{-i h_{\mathrm{B}} t / \hbar}$ acts on the system. As exact theory is concerned, the bath correlation function $C(t-\tau) \equiv\left\langle F_{\mathrm{B}}(t) F_{\mathrm{B}}(\tau)\right\rangle$ is related to the bath spectral density function $J(\omega)$ via

$$
C(t)=\frac{1}{\pi} \int_{-\infty}^{\infty} d \omega e^{-i \omega t} \frac{J(\omega)}{1-e^{-\beta \omega}} .
$$

This is the fluctuation-dissipation theorem of bosonic canonical ensembles, where $\beta \equiv \hbar /\left(k_{B} T\right)$ denotes the inverse temperature.

\footnotetext{
*Electronic address: rxxu@ustc.edu.cn

${ }^{\dagger}$ Electronic address: yyan@ust.hk
}

The common strategy of memory decomposition used for HEOM construction is based on the Matsubara series expansion of Bose (also called Bose-Einstein) function in Eq. (11). It assumes (setting $x=\beta \omega$ )

$$
\frac{1}{1-e^{-x}} \approx \frac{1}{2}+\frac{1}{x}+\sum_{m=1}^{N}\left(\frac{1}{x+i 2 \pi m}+\frac{1}{x-i 2 \pi m}\right)
$$

This is the Matsubara spectral decomposition (MSD) scheme. It is exact when $N \rightarrow \infty$. It resolves the memory contents of bath correlation function with Matsubara frequencies $(2 \pi m / \beta ; m \geq 1)$, together with the poles of $J(z)$ in lower plane $(\operatorname{Im} z<0)$. In practical use, the residue $\delta C(t)$, due to the deviation of Eq. (2) from the exact one, is treated by white noise [13]. The resulting HEOM formalism has been applied to electron/population transfer and optical spectroscopy systems [14, 15, 16, 17].

In this work, we explore the partial fraction decomposition (PFD) scheme. This scheme was originally proposed for Fermi function in the study of electronic dynamics/structure systems [18, 19, 20, 21, 22, 23, 24]. The motivation behind is that MSD is well-known to be of slow convergence. The PFD of Bose function will be carried out in a similar manner as that proposed recently by Croy and Saalmann for the Fermi function 24]. We present the PFD results on Bose function in Sec.II and the corresponding HEOM-QDT construction in Sec.III Their derivations are given in Appendix $\mathrm{A}$ and Appendix $\mathrm{B}$, respectively.

Numerical performance of the new HEOM-QDT will be exemplified with a model spin-boson Drude dissipation system in Sec.IV. Included are also comparisons with the best available MSD-based conventional HEOM theory. The latter is implemented with the wellestablished Markovian residue correction method [6, 7] that has improved significantly the performance of MSDbased HEOM. Nevertheless, the PFD-based HEOM theory without residue correction still performs much better, about 20 times faster, than the best available MSD-based approach. Finally we conclude this work. 

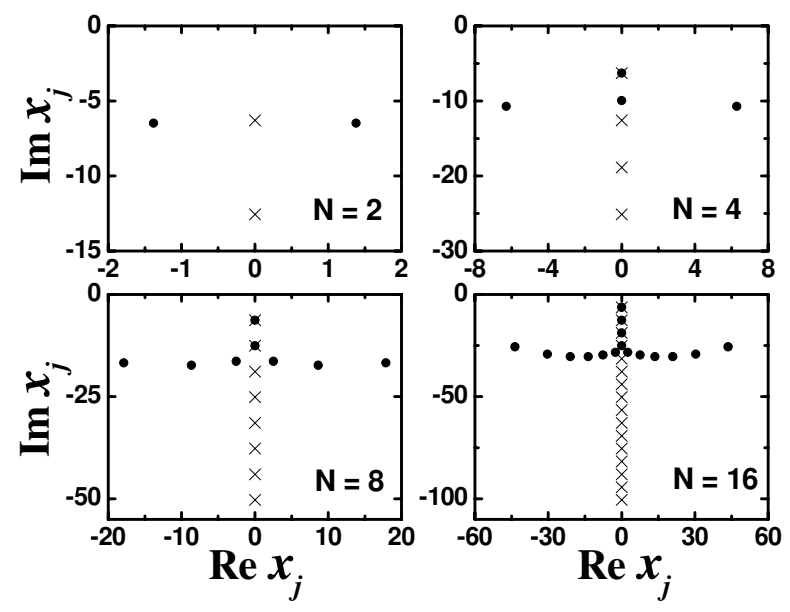

FIG. 1: Poles of the PFD expansion (solid circle) at the expansion order $N=2,4,8,16$. Included for comparison are also the Matsubara poles (cross). Poles are complex-conjugate paired, and only those in lower plane are shown.

\section{PARTIAL FRACTION DECOMPOSITION SCHEME}

\section{A. Bose-Einstein function in decomposition}

The PFD scheme starts with the identity

$$
\frac{1}{1-e^{-x}}=\frac{1}{2}+\frac{1}{2} \frac{\cosh (x / 2)}{\sinh (x / 2)}
$$

followed by Taylor's expansions of the numerator function $\cosh (x / 2)$ to the $(2 N)^{\text {th }}$-order and the denominator $\sinh (x / 2)$ to the $(2 N+1)^{\text {th }}-$ orders, respectively. PFD leads to Bose function the expression (cf. Appendix A)

$$
\frac{1}{1-e^{-x}} \approx \frac{1}{2}+\frac{1}{x}+\sum_{j=1}^{N}\left(\frac{1}{x+2 \sqrt{\xi_{j}}}+\frac{1}{x-2 \sqrt{\xi_{j}}}\right) .
$$

The involving parameters $\left\{\xi_{j} ; j=1, \cdots N\right\}$ are the roots of the denominator polynomial, $\sum_{n=0}^{N} \xi^{n} /(2 n+1) !=0$. They can be determined as the eigenvalues of an $N \times N$ matrix whose elements are

$$
A_{m n}=2 m(2 m+1) \delta_{m+1, n}-2 N(2 N+1) \delta_{m N} .
$$

Note that the Fermi function counterpart is similar [24], just replacing +1 with -1 in both parentheses in Eq. (5). The derivation of the above formalism is detailed in Appendix A

Figure 1 depicts the poles $x_{j} \equiv \pm 2 \sqrt{\xi_{j}}$ of the PFD scheme at different orders. Shown are only those $N$ poles in lower plane, and their complex conjugates (in upper plane) are implied. The distribution pattern of the total $2 N$ poles is similar to that of Fermi function [24]. There are not only pure imaginary poles, which are mostly close to the Matsubara poles, but also complex

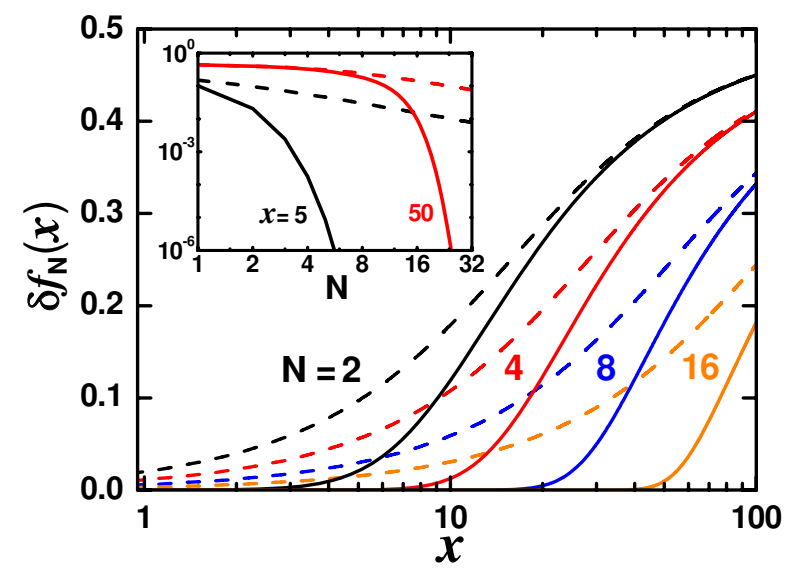

FIG. 2: The difference $\delta f_{N}(x) \equiv f_{\mathrm{BE}}(x)-f_{N}(x)$, between the exact Bose-Einstein function and its PFD expansion approximation [Eq. (4)], as the function of $x$ at $N=2$ (black), 4 (red), 8 (blue), and 16 (orange). The inset is for $\delta f_{N}(x)$ as function of $N$, at $x=5$ (black) and 50 (red). The Matsubara counterparts are also shown by dashed curves.

poles with nonzero real parts. This is right the feature for the efficiency of PFD scheme.

Figure 2 depicts the deviation of the approximations from the exact Bose function. The curves there can be used to estimate the required order of $N$ for the PFDHEOM dynamics, by considering the effective system and bath frequencies. It is clearly seen that the PFD expansion well overlaps with the exact result, within the range $|x|<4 N$ (i.e., $|\omega|<4 N k_{B} T / \hbar$ ), as estimated, although for the case of Fermi function by Croy and Saalmann recently [24].

\section{B. Correlation function in decomposition}

For the later construction of HEOM formalism, we shall expand the bath correlation function $C(t)$ in an exponential series [9]. This can be achieved via contour integration method. It recasts Eq. (11) via analytic continuation as

$$
C(t)=\frac{1}{\pi} \oint d z e^{-i z t} \frac{J(z)}{1-e^{-\beta z}},
$$

and evaluates it by Cauchy's residue theorem. The contour of integration encloses the lower-half plane for the required $C(t>0)$. Denote

$$
C(t) \equiv C_{0}(t)+C_{\mathrm{B}}(t),
$$

for the contributions from the poles of spectral density $J(z)$ and Bose function, respectively. Note that the antisymmetry property of bosonic spectral density, $J(-\omega)=-J(\omega)$, reads now

$$
J\left(-z^{*}\right)=-J^{*}(z) .
$$


Consider first the Bose function contribution $C_{\mathrm{B}}(t)$. The total $N$ PFD poles in lower plane are either pure imaginary or true complex numbers in pairs, $\left\{z_{j},-z_{j}^{*}\right\}$, as depicted in Fig.11. With separation of pure imaginary and true complex values, we denote these $N$ poles as

$$
z_{s}=\left\{\begin{array}{cl}
-i \gamma_{s} ; & s=1, \cdots, N_{r} \\
-\left(\omega_{s}+i \gamma_{s}\right) ; & s=N_{r}+1, \cdots, N_{p}
\end{array},\right.
$$

together with $-z_{s}^{*}=\omega_{s}-i \gamma_{s}$ in lower plane; thus, $N_{p} \equiv$ $\left(N+N_{r}\right) / 2$. The parameters $\gamma_{s}$ and $\omega_{s}$ are all positive. The corresponding residues are (Res) $z_{s}=e^{-i z_{s} t} J\left(z_{s}\right)$ and $(\operatorname{Res})_{\left(-z_{s}^{*}\right)}=e^{i z_{s}^{*} t} J\left(-z_{s}^{*}\right)=-\left[(\operatorname{Res})_{z_{s}}\right]^{*}$. The last identity is inferred from Eq. (8). We have therefore

$C_{\mathrm{B}}(t)=\sum_{s=1}^{N_{r}} c_{s} e^{-\gamma_{s} t}+\sum_{s=N_{r}+1}^{N_{p}} e^{-\gamma_{s} t}\left[a_{s} \cos \left(\omega_{s} t\right)-b_{s} \sin \left(\omega_{s} t\right)\right]$.

It decomposes $C_{\mathrm{B}}(t)$ into a total of $N$ components. The involving coefficients are all real and defined as

$$
c_{s} \equiv \frac{2}{i \beta} J\left(-i \gamma_{s}\right), \quad a_{s}+i b_{s} \equiv \frac{4}{i \beta} J\left[-\left(\omega_{s}+i \gamma_{s}\right)\right] .
$$

The fact that $c_{s}$ is real can be readily proved via its definition and Eq. (8). Thus, $C_{\mathrm{B}}(t)$ is always a real function.

The $C_{0}(t)$ component of bath correlation function, which is complex in general, arises from the poles of spectral density $J(z)$. In principle it would not depend on $\mathrm{PFD} / \mathrm{MSD}$ scheme; but in practice it will, for the issue of consistency to be discussed later. In the following HEOM construction, we consider the Drude model,

$$
J(\omega)=\frac{\eta \omega_{c} \omega}{\omega^{2}+\omega_{c}^{2}},
$$

where $\eta$ is the system-bath coupling strength and $\omega_{c}$ the cut-off frequency. The Drude spectral density function has only one pole in lower plane. It results in

$$
C_{0}(t)=c_{0} e^{-\omega_{c} t},
$$

with

$$
c_{0}=-i \eta \omega_{c}\left(\frac{1}{1-e^{-\beta z}}\right)_{z=-i \omega_{c}} .
$$

The above $C_{0}(t)$ expression is exact. However, a consistency issue arises as the Bose function used in evaluating $C_{\mathrm{B}}(t)$ via Eq. (10) involves the PFD approximation. The inconsistency here may be problematic in implementation. For example, the divergence of the above exact $C_{0}(t)$ at $\beta \omega_{c}=2 \pi m$ cannot be cancelled out by the approximated $C_{\mathrm{B}}(t)$ of Eq. (10). To overcome this problem, we evaluate $c_{0}$ also using PFD of Eq. (4) with the same finite $N$.

$$
c_{0}=-i \eta \omega_{c}\left(\frac{1}{2}+\frac{1}{\beta z}+\frac{1}{\beta} \sum_{j=1}^{N} \frac{2 z}{z^{2}-z_{j}^{2}}\right)_{z=-i \omega_{c}} .
$$

The resulting $C_{0}(t)$ does practically depend on the scheme of expansion for Bose function. We shall emphasize the importance of the aforementioned consistent treatment between $C_{0}(t)$ and $C_{\mathrm{B}}(t)$. We have recently demonstrated it with different approximation schemes [17. We have also confirmed this issue with the present PFD scheme, not just for $C(t)=C_{0}(t)+C_{\mathrm{B}}(t)$, but more importantly for the numerical performance of the resulting HEOM dynamics to be presented below.

\section{HIERARCHICAL EQUATIONS OF MOTION}

\section{A. HEOM-QDT in PFD scheme}

The generic form of HEOM-QDT reads [9]

$$
\dot{\rho}_{\mathrm{n}}=-\left(i \mathcal{L}+\Gamma_{\mathrm{n}}\right) \rho_{\mathrm{n}}+\rho_{\mathrm{n}}^{\{\leftrightarrow\}}+\rho_{\mathrm{n}}^{\{-\}}+\rho_{\mathrm{n}}^{\{+\}} .
$$

Here, $\mathcal{L} \cdot \equiv \hbar^{-1}[H, \cdot]$ is the reduced system Liouvillian; $\Gamma_{\mathrm{n}}$ is the decay constant; $\rho_{\mathrm{n}}, \rho_{\mathrm{n}}^{\{\leftrightarrow\}}, \rho_{\mathrm{n}}^{\{-\}}$, and $\rho_{\mathrm{n}}^{\{+\}}$are welldefined auxiliary density operators (ADOs) in the system subspace. The reduced system density operator of primary interest, defined as $\rho(t) \equiv \operatorname{Tr}_{\text {bath }} \rho_{\text {total }}(t)$, is just $\rho(t) \equiv \rho_{0}(t)$, with $\rho_{0}^{\{\leftrightarrow\}}=\rho_{0}^{\{-\}}=\Gamma_{0}=0$. The labeling index $\mathrm{n}$ for a generic $\mathrm{ADO} \rho_{\mathrm{n}}$ consists of a set of nonnegative integers, which are arranged in relation to the individual components of bath correlation function in a given decomposition scheme. Let $\mathrm{n}=\left\{n_{0}, n_{1}, \cdots, n_{N}\right\}$ and $n_{0}+n_{1}+\cdots+n_{N}=\tilde{n}$. The latter is used to define the tier of $\rho_{\mathrm{n}}$. The last three terms in Eq. (15) describes how a given $\rho_{\mathrm{n}}$ of $\tilde{n}^{\text {th }}$ tier depends on its associated ADOs of same tier $\left(\rho_{\mathrm{n}}^{\{\leftrightarrow\}}\right)$ and neighboring tiers $\left(\rho_{\mathrm{n}}^{\{ \pm\}}\right)$, respectively.

The HEOM-QDT formalism is exact and nonperturbative, assuming only Gaussian bath statistics. It is equivalent to the Feynman-Vernon path integral influence functional theory of reduced system density operator dynamics. Moreover, it also supports the incorporation of the initial system-bath correlation through appropriate initial $\rho_{\mathbf{n} \neq 0}\left(t_{0}\right) \neq 0$ conditions. HEOM resolves the combined effect of the coupling bath strength and memory contents, as they are decomposed, on the reduced system dynamics.

The specific form of theory depends on the way of decomposing the bath correlation function $C(t)$. For Drude dissipation in the PFD scheme, $C(t)$ [Eq. (7)] has been decomposed into $(N+1)$ components in Eqs. (10) and (13). Therefore, the ADO labeling index $n$ assumes

$$
\mathrm{n}=\left\{n_{s=0,1, \cdots, N_{r}} ; n_{s=N_{r}+1, \cdots, N_{p}} ; \bar{n}_{s=N_{r}+1, \cdots, N_{p}}\right\} .
$$

The composite $(N+1)$ nonnegative integers are the leading orders of individual components of $C(t)$, involved in the specified $\rho_{\mathrm{n}}$. Specifically, $n_{0}$ is for the Drude component $C_{0}(t)$ of Eq. (13), and the other $N$ integers are for the $N$ components of $C_{\mathrm{B}}(t)$ of Eq. (10), respectively.

In Appendix B, we present the standard approach to the corresponding HEOM formalism, based on the present PFD scheme. The final results are summarized as 
follows. The parameter $\Gamma_{\mathrm{n}}$ in Eq. (15) collects all damping factors. It reads (setting $\left.\gamma_{0} \equiv \omega_{c}\right)$ :

$$
\Gamma_{\mathrm{n}}=\sum_{s=0}^{N_{r}} n_{s} \gamma_{s}+\sum_{s=N_{r}+1}^{N_{p}}\left(n_{s}+\bar{n}_{s}\right) \gamma_{s}
$$

It arises from the derivatives of individual exponential components of $C(t)$, involved in $\rho_{\mathrm{n}}$.

The swap term $\rho_{\mathrm{n}}^{\{\leftrightarrow\}}$ describes how a given $\rho_{\mathrm{n}}$ depends on its associated ADOs of same tier. It reads

$$
\begin{array}{r}
\rho_{\mathrm{n}}^{\{\leftrightarrow\}}=\sum_{s=N_{r}+1}^{N_{p}} \omega_{s}\left[\left(a_{s} / b_{s}\right) \sqrt{n_{s}\left(\bar{n}_{s}+1\right)\left|b_{s} / a_{s}\right|} \rho_{\mathrm{n}_{s}}\right. \\
\left.-\left(b_{s} / a_{s}\right) \sqrt{\bar{n}_{s}\left(n_{s}+1\right)\left|a_{s} / b_{s}\right|} \rho_{\mathrm{n}_{s}}\right],
\end{array}
$$

It arises from the derivatives of the sine and cosine components of $C_{\mathrm{B}}(t)$ [Eq. (10)] involved in $\rho_{\mathrm{n}}$. The involving index $\mathrm{n}_{s}^{\rightarrow}$ differs from $\mathrm{n}$ by changing $\left(n_{s}, \bar{n}_{s}\right)$ to $\left(n_{s}-1, \bar{n}_{s}+1\right)$ and $\mathrm{n}_{s}^{\leftarrow}$ by changing $\left(n_{s}, \bar{n}_{s}\right)$ to $\left(n_{s}+1, \bar{n}_{s}-1\right)$.

The last two terms in Eq. (15) describes the tier-down and tier-up contributions. They are given by

$$
\begin{aligned}
& \rho_{\mathrm{n}}^{\{-\}}=-\frac{i}{\hbar} \sum_{s=0}^{N_{r}} \sqrt{n_{s} /\left|c_{s}\right|}\left(c_{s} Q \rho_{\mathrm{n}_{s}^{-}}-c_{s}^{*} \rho_{\mathrm{n}_{s}^{-}} Q\right) \\
& -\frac{i}{\hbar} \sum_{s=N_{r}+1}^{N_{p}} a_{s} \sqrt{n_{s} /\left|a_{s}\right|}\left[Q, \rho_{\mathrm{n}_{s}^{-}}\right] \text {, } \\
& \rho_{\mathrm{n}}^{\{+\}}=-\frac{i}{\hbar} \sum_{s=0}^{N_{r}} \sqrt{\left(n_{s}+1\right)\left|c_{s}\right|}\left[Q, \rho_{\mathrm{n}_{s}^{+}}\right] \\
& -\frac{i}{\hbar} \sum_{s=N_{r}+1}^{N_{p}}\left\{\sqrt{\left(n_{s}+1\right)\left|a_{s}\right|}\left[Q, \rho_{\mathrm{n}_{s}^{+}}\right]\right. \\
& \left.+\sqrt{\left(\bar{n}_{s}+1\right)\left|b_{s}\right|}\left[Q, \rho_{\overline{\mathrm{n}}_{s}^{+}}\right]\right\} .
\end{aligned}
$$

They depend explicitly on the system dissipation mode $Q$. The involving index $\mathrm{n}_{s}^{ \pm}$differs from $\mathrm{n}$ by changing the specified $n_{s}$ to $n_{s} \pm 1$, and $\overline{\mathrm{n}}_{s}^{ \pm}$similarly.

Apparently, the boundary conditions of $\rho_{0}^{\{\leftrightarrow\}}=\rho_{0}^{\{-\}}=$ $\Gamma_{0}=0$ are satisfied. All coefficients/parameters arising from the Bose part $C_{\mathrm{B}}(t)$ of bath correlation, $\left\{a_{s}, b_{s}, c_{s}, \gamma_{s}, \omega_{s}\right\}_{s>0}$, are real; the last two are positive. The Drude damping parameter is set to be $\gamma_{0}=\omega_{c}$. Only $c_{0}[\mathrm{Eq} \cdot(14)]$ is complex.

\section{B. Remarks on implementation}

To facilitate locating a specified ADO, we also like to have a working index scheme to map a set of $(N+1)$ ordered multiple indices, $\mathrm{n} \equiv\left\{n_{0}, n_{1}, \cdots, n_{N}\right\}$, to an integer $j_{\mathrm{n}}$, such that $\rho_{\mathrm{n}} \equiv \rho_{j_{\mathrm{n}}}$. That $\rho_{\mathrm{n}}$ is an $\tilde{n}^{\text {th }}-$ tier
ADO if its $n_{0}+\cdots+n_{N} \equiv \tilde{n}$. The number of ADOs at a given tier is $\frac{(N+\tilde{n}) !}{N ! \tilde{n} !}$, while the total number of ADOs up to level $L$ is

$$
\mathcal{N}=\sum_{\tilde{n}=0}^{L} \frac{(N+\tilde{n}) !}{N ! \tilde{n} !} \equiv\left\{\begin{array}{c}
L \\
N
\end{array}\right\}
$$

The second identity serves also the definition of $\left\{\begin{array}{c}m \\ n\end{array}\right\}$ for abbreviated notion, with the boundary values of $\left\{\begin{array}{c}m<0 \\ n\end{array}\right\}=0$ and $\left\{\begin{array}{c}0 \\ n\end{array}\right\}=\left\{\begin{array}{c}m \\ 0\end{array}\right\}=1$. The working index $j_{\mathrm{n}} \equiv j_{n_{0} \cdots n_{N}}=0, \cdots, \mathcal{N}-1$ can then be

$$
j_{\mathrm{n}}=\left\{\begin{array}{c}
\tilde{n}-1 \\
N
\end{array}\right\}+\sum_{q=0}^{N}\left\{\begin{array}{c}
n_{q+1}+\cdots+n_{N}-1 \\
N-q
\end{array}\right\} .
$$

It sorts the index $\mathrm{n}$ by tiers and followed by subindices, so that $j_{\mathrm{n}=\{0, \cdots, 0\}}=0, j_{\mathrm{n}=\{1,0, \cdots, 0\}}=1, \cdots$, and $j_{\mathrm{n}=\{0, \cdots, 0, L\}}=\mathcal{N}-1$.

In practice, HEOM should be truncated properly at finite level of hierarchy $L$ and decomposition order $N$. By far the truncation of $N$ goes with convergency, but that of $L$ are carried out effectively and automatically. Apparently, the issue of truncation is closely related to central processing unit (CPU) time and memory cost of computation. The number $\mathcal{N}$ of total ADOs goes with a combinatory law, like the complete configuration interaction treatment in quantum mechanics. Shi et al. have recently proposed an efficient, accuracy-controlled, dynamics filtering algorithm [13, 14]. The basic idea behind is the observation that only a very small fraction of total ADOs play roles in HEOM propagation. The filtering algorithm sets a specific $\rho_{\mathrm{n}}\left(t_{j}\right)=0$ if its matrix elements amplitudes are all smaller than the pre-chosen error tolerance. To validate this simple algorithm, all ADOs including the reduced density matrix of the primary interest should have a uniform filtering-algorithm error tolerance. The present HEOM formalism [Eqs. (15)-(20)] has been scaled properly to meet this requirement. In connection with the stochastic field approach to GaussianMarkovian dissipation, the scaled ADOs are just the expansion coefficients, with the normalized harmonic wave functions used as the basis set for resolving the diffusive bath field [14]. The filtering algorithm keeps only those necessary ADOs according to the selected error tolerance. Apparently, it also automatically truncates the level of hierarchy on-the-fly during numerical propagation.

\section{NUMERICAL PERFORMANCE AND CONCLUDING REMARKS}

The performance of the present PFD-based HEOM dynamics is exemplified with Fig. 3 for a spin-boson system $H=\epsilon \sigma_{z}+\Delta \sigma_{x}$, with the dissipation mode of $Q=\sigma_{z}$. The parameters for the system and coupling bath spectral density are $\left(\epsilon, \eta, \omega_{c}\right)=(1,0.5,5)$ in unit of $\Delta$, with $\Delta=1660 \mathrm{~cm}^{-1}$ and $T=298 \mathrm{~K}$. The result of PFDHEOM with $N=12$ in Fig. 3 is practically converged, 


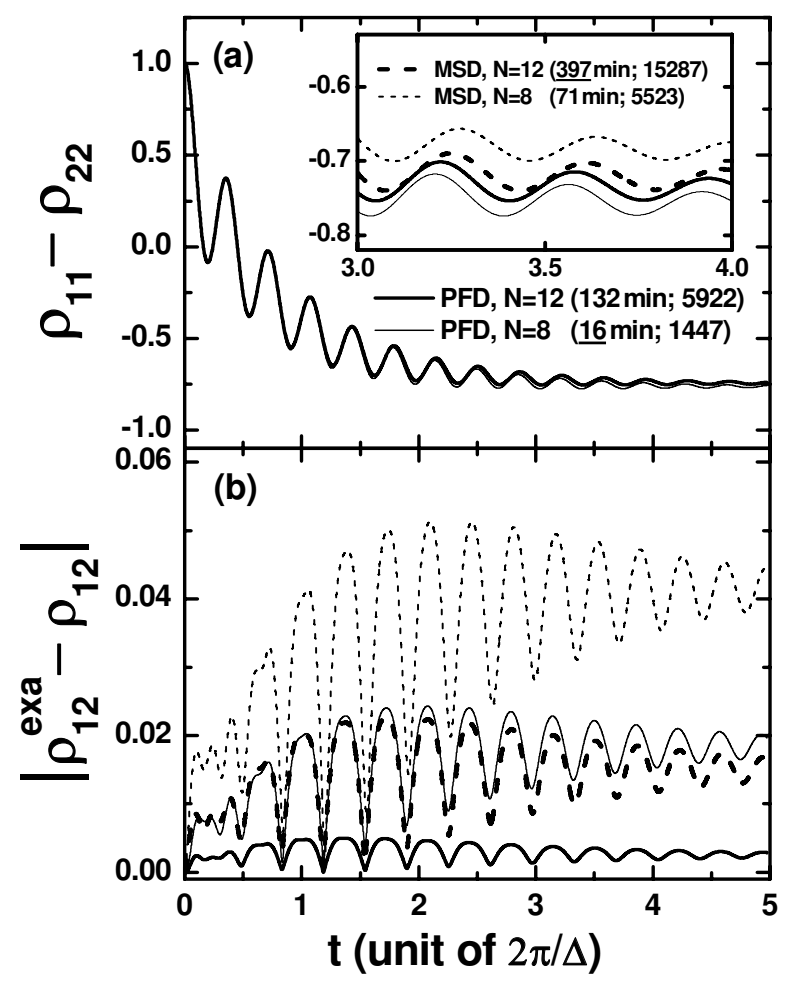

FIG. 3: Time evolution of a spin-boson system density matrix: (a) the population difference $\rho_{11}(t)-\rho_{22}(t)$; (b) the deviation of off-diagonal $\rho_{12}(t)$ from the exact. The initial conditions are all $\rho_{\mathrm{n}}(0)=0$; except for $\rho_{\mathrm{n}=0}(0)=\rho(0)$ where $\rho_{11}(0)=1$ and $\rho_{22}(0)=\rho_{12}(0)=0$. The specified values of (CPU time; $\left.\mathcal{N}_{\text {eff }}\right)$ highlight the implementation cost. Especially the two underlined values indicate that PFD-HEOM converges about 25 times faster than MSD-HEOM. See text for details.

within 0.005 from that with $N=16$. The latter is treated as the exact in Fig.3(b). The maximum tier of survival ADOs is found to be $L_{\max }=8$, with the filtering algorithm error tolerance of $10^{-7}$; see Sec.IIIB or Ref. [13].

Included for comparison are also the results of the best available MSD-based conventional HEOM theory. This reference theory is augmented with the well-established Markovian residue correction (MRC) [6, 7], in which $\delta C(t) \equiv C_{\text {exact }}(t)-C_{\mathrm{MSD}}(t)$ is approximated by white noise for its effect on the MSD-based HEOM dynamics. Without additional implementation cost, this treatment significantly reduces the required number $N$ for converged MSD dynamics [6, 7, 13, 14, 15, 16, 17]. This remarkable MSD-based feature remains true for the system in Fig. 3, However, MRC does not work well with the present PFD-HEOM theory. Therefore the comparison shown in Fig. [3] is between PFD-HEOM without residue correction versus MSD-HEOM with MRC, the best available reference as we know.

Performance is reported in terms of (CPU time; $\left.\mathcal{N}_{\text {eff }}\right)$. The CPU time is by a single $\operatorname{Intel}(\mathrm{R}) \mathrm{Xeon}(\mathrm{R})$ processor @3.00 GHz, with the fourth-order Runge-Kutta propagator and time-step of $0.0015 \mathrm{fs}$. Another parameter
$\mathcal{N}_{\text {eff }}$ records the largest number of ADOs ever survived during propagation with the filtering algorithm. Recall that the ADOs in PFD-HEOM is of the maximum survival tier level of $L_{\max }=8$. In contrast, a converged MSD-HEOM dynamics requires the level of about 20 tiers.

For the purpose of comparison, however, we set $L=$ 9 in all calculations, including both PFD and MSD schemes. Apparently, $\mathcal{N}_{\text {eff }}$ in either scheme is orders of magnitude smaller than the total number of ADOs, which is $\mathcal{N}=497420$ for $N=12$ or $\mathcal{N}=48620$ for $N=8$, respectively. The PFD-HEOM is of smaller $\mathcal{N}_{\text {eff }}$, about $25 \sim 40 \%$ of its MSD counterpart with same $L$ and $N$. The enhanced filtering efficiency in PFD scheme here is closely related to its complex poles, rather than only pure imaginary ones (Fig.11). The complex poles lead to oscillatory decomposition components of bath correlation function [Eq. (10)], and result in oscillatory cancelation in PFD-HEOM dynamics. This right property of the PFD scheme may account for the relatively small number of survival ADOs after filtering algorithm.

Performance of PFD-HEOM dynamics should be based on the CPU time versus its MSD counterpart of the same quality. As mentioned earlier, however, the converged MSD dynamics for the present system of study is too expensive to be worth here. We rather choose for assessment by a pair of similar quality but approximated results: $\operatorname{PFD}(N=8)$ versus $\operatorname{MSD}(N=12)$, with the CPU time of 16 minutes versus 397 minutes, respectively, see Fig.3. We have carried out a series of dynamics simulations with different system and bath parameters. All results show that the performance of PFD-HEOM is superior over its MSD counterpart by at least an order of magnitude.

In summary, we have constructed the PFD scheme to Bose function and HEOM-QDT. The superiority of PFD over MSD is apparent. The complex PFD poles lead to not just the Bose function expansion more efficient and accurate, but also the HEOM construction more compact. The resulting HEOM dynamics converges with smaller $L$ and $N$, and also accommodates better with the propagation filtering algorithm. These factors contribute the performance of PFD-based HEOM theory, which converges a magnitude (about 20 times) faster than its MSD counterpart.

\section{Acknowledgement}

Support from the National Natural Science Foundation of China (20533060 and 20773114), National Basic Research Program of China (2006CB922004), and RGC Hong Kong (604508 and 604709) is acknowledged. 


\section{APPENDIX A: DERIVATION OF BOSE FUNCTION IN PFD EXPANSION}

The derivation of Eqs. (41) and (5) starts with the Taylor's expansions $\cosh (y) \approx \sum_{n=0}^{N} y^{2 n} /(2 n)$ ! and $\sinh (y) \approx \sum_{n=0}^{N} y^{2 n+1} /(2 n+1)$ ! for the numerator and denominator of the last term in Eq. (3), respectively. We have then

$$
\frac{1}{1-e^{-x}} \approx \frac{1}{2}+\frac{1}{x}+B_{N}(x / 2) .
$$

where

$$
\begin{aligned}
B_{N}(y) & =\frac{1}{2} \frac{\sum_{n=0}^{N} y^{2 n} /(2 n) !}{\sum_{n=0}^{N} y^{2 n+1} /(2 n+1) !}-\frac{1}{2 y} \\
& =\frac{\sum_{n=1}^{N} \frac{n}{(2 n+1) !} y^{2 n-1}}{\sum_{n=0}^{N} \frac{1}{(2 n+1) !} y^{2 n}} \\
& \equiv \frac{1}{2} \sum_{j=1}^{N}\left(\frac{b_{j}^{+}}{y+\sqrt{\xi_{j}}}+\frac{b_{j}^{-}}{y-\sqrt{\xi_{j}}}\right),
\end{aligned}
$$

with $\left\{y_{j}= \pm \sqrt{\xi_{j}} ; j=1, \cdots, N\right\}$ being the roots of the denominator polynomial; i.e.,

$$
\sum_{n=0}^{N} \frac{1}{(2 n+1) !} \xi^{n}=0 .
$$

The PFD coefficient $b_{j}^{ \pm}$can be determined via

$$
\begin{aligned}
b_{j}^{ \pm} & =2 \lim _{y \pm \sqrt{\xi_{j}}=0}\left[\left(y \pm \sqrt{\xi_{j}}\right) B_{N}(y)\right] \\
& =2 \lim _{y=0}\left[y B_{N}\left(y \mp \sqrt{\xi_{j}}\right)\right] \\
& =\left.\frac{\left[2 y \sum_{n=1}^{N} \frac{n}{(2 n+1) !}\left(y \mp \sqrt{\xi_{j}}\right)^{2 n-1}\right]^{\prime}}{\left[\sum_{n=0}^{N} \frac{1}{(2 n+1) !}\left(y \mp \sqrt{\xi_{j}}\right)^{2 n}\right]^{\prime}}\right|_{y=0} \\
& =\frac{\sum_{n=1}^{N} \frac{2 n}{(2 n+1) !}\left(\mp \sqrt{\xi_{j}}\right)^{2 n-1}}{\sum_{n=0}^{N} \frac{2 n}{(2 n+1) !}\left(\mp \sqrt{\xi_{j}}\right)^{2 n-1}} \\
& =1 .
\end{aligned}
$$

The PFD expression of Bose function, Eq. (4), is obtained by substituting Eqs. (A2) and (A4) into Eq. (A1), with $x=2 y$.

To convert the roots of polynomial to eigenvalue problem, as Eq. (5), let us consider the general case of

$$
a_{0}+a_{1} \xi+\cdots+a_{N} \xi^{N}=0 .
$$

We search for the proper matrix $\boldsymbol{A} \equiv\left\{A_{m n}\right\}$, with the eigenvector the form of $\mathbf{v}=\left[c_{1}, c_{2} \xi, \cdots, c_{N} \xi^{N-1}\right]^{T}$ that converts the eigenequation $\boldsymbol{A v}=\xi \mathbf{v}$ to

$$
\sum_{n=1}^{N} A_{m n} c_{n} \xi^{n-1}=c_{m} \xi^{m} ; \quad m=1, \cdots, N .
$$

By comparing Eqs. (A5) and (A6) and setting $c_{N}=$ $a_{N}$, we have the following nonzero elements: $A_{N n}=$ $-a_{n-1} / c_{n}$ and $A_{m, m+1}=c_{m} / c_{m+1}$ for $m \neq N$; others are all zeroes. Therefore,

$$
A_{m n}=\frac{c_{m}}{c_{m+1}} \delta_{m+1, n}-\frac{a_{n-1}}{c_{n}} \delta_{m N},
$$

with $c_{n}$ being arbitrary nonzero parameters, except for $c_{N}=a_{N}$.

In particular, we choose $c_{n}=a_{n-1} a_{N} / a_{N-1}$, if every $a_{j} \neq 0$. The boundary condition of $c_{N}=a_{N}$ is also satisfied. We can recast Eq. (A7) as

$$
A_{m n}=\frac{a_{m-1}}{a_{m}} \delta_{m+1, n}-\frac{a_{N-1}}{a_{N}} \delta_{m N} .
$$

It recovers Eq. (5) for the polynomial Eq. (A3), where $a_{n}=1 /(2 n+1) !$.

\section{APPENDIX B: DERIVATION OF HEOM-QDT IN PFD SCHEME}

The following derivation of the HEOM in Sec.IIIA is carried out via the well-established Calculus-On-PathIntegral Influence-Generating-Functional (COPI-IGF) algebra [9]. It starts with the path integral influence functional for quantum Gaussian dissipation [1, 2, 3], followed by consecutive time derivatives to resolve in a hierarchical manner the involving memory contents [4, 5, 8]. Unlike the HEOM theory that can be expressed in operator level, the path integral formalism has to go with representation. Let $\{|\alpha\rangle\}$ be a generic basis set in the system subspace and $\boldsymbol{\alpha} \equiv\left(\alpha, \alpha^{\prime}\right)$ for abbreviation, such that $\rho(\boldsymbol{\alpha}, t) \equiv \rho\left(\alpha, \alpha^{\prime}, t\right) \equiv\left\langle\alpha|\rho(t)| \alpha^{\prime}\right\rangle$. Introduce the reduced Liouville-space propagator $\mathcal{U}$ via

$$
\rho(\boldsymbol{\alpha}, t) \equiv \int d \boldsymbol{\alpha}_{0} \mathcal{U}\left(\boldsymbol{\alpha}, t ; \boldsymbol{\alpha}_{0}, t_{0}\right) \rho\left(\boldsymbol{\alpha}_{0}, t_{0}\right) .
$$

Its path-integral expression reads

$$
\mathcal{U}\left(\boldsymbol{\alpha}, t ; \boldsymbol{\alpha}_{0}, t_{0}\right)=\int_{\boldsymbol{\alpha}_{0}\left[t_{0}\right]}^{\boldsymbol{\alpha}[t]} \mathcal{D} \boldsymbol{\alpha} e^{i S[\alpha] / \hbar} \mathcal{F}[\boldsymbol{\alpha}] e^{-i S\left[\alpha^{\prime}\right] / \hbar} .
$$

$S$ and $\mathcal{F}$ are the action and influence functionals, respectively. For Gaussian bath interactions, the latter assumes the Feynman-Vernon form that can be recast as [1, 9]

$$
\mathcal{F}[\boldsymbol{\alpha}]=\exp \left\{-\frac{i}{\hbar} \int_{t_{0}}^{t} d \tau \mathcal{A}[\boldsymbol{\alpha}(\tau)] \mathcal{B}(\tau ;\{\boldsymbol{\alpha}\})\right\},
$$

where

$$
\mathcal{A}[\boldsymbol{\alpha}(t)]=Q[\alpha(t)]-Q\left[\alpha^{\prime}(t)\right],
$$

$$
\mathcal{B}(t ;\{\boldsymbol{\alpha}\})=-\frac{i}{\hbar}\left[B(t ;\{\alpha\})-B^{\prime}\left(t ;\left\{\alpha^{\prime}\right\}\right)\right]
$$


with

$$
\begin{aligned}
B(t ;\{\alpha\}) & \equiv \int_{t_{0}}^{t} d \tau C(t-\tau) Q[\alpha(\tau)], \\
B^{\prime}\left(t ;\left\{\alpha^{\prime}\right\}\right) & \equiv \int_{t_{0}}^{t} d \tau C^{*}(t-\tau) Q\left[\alpha^{\prime}(\tau)\right] .
\end{aligned}
$$

Note that $\mathcal{A}[\boldsymbol{\alpha}(t)]$ depends only on the local time of path and assumes readily in operator level as commutator, $\mathcal{A} \cdot=[Q, \cdot]$. In contrast, the functional $\mathcal{B}(t ;\{\alpha\})$ contains memory and does not have a simple operatorlevel correspondence. The COPI-IGF algebra provides a proper hierarchy to resolve the memory contents involved in $\mathcal{B}(t ;\{\alpha\})$.

To proceed, we decompose $\mathcal{B}(t ;\{\alpha\})$ according to the decomposition of bath correlation function $C(t)$ [Eq. (77) with Eqs. (10) and (13)]. We have

$$
\mathcal{B}=\sum_{s=0}^{N_{r}} \mathcal{B}_{s}+\sum_{s=N_{r}+1}^{N_{p}}\left(\mathcal{B}_{s}+\overline{\mathcal{B}}_{s}\right)
$$

where (noting that $c_{0}$ is complex while others are real)

$$
\begin{array}{rlrl}
\mathcal{B}_{s} & \equiv-\frac{i}{\hbar}\left(c_{s} B_{s}-c_{s}^{*} B_{s}^{\prime}\right) ; & s=0,1, \cdots, N_{r} \\
\mathcal{B}_{s} & \equiv-\frac{i}{\hbar} a_{s}\left(B_{s}-B_{s}^{\prime}\right) ; & s & =N_{r}+1, \cdots, N_{p} \\
\overline{\mathcal{B}}_{s} & \equiv \frac{i}{\hbar} b_{s}\left(\bar{B}_{s}-\bar{B}_{s}^{\prime}\right) ; & s & =N_{r}+1, \cdots, N_{p} .
\end{array}
$$

with (denoting $\omega_{s \leq N_{r}} \equiv 0$ )

$$
\begin{aligned}
& B_{s}=\int_{t_{0}}^{t} d \tau e^{-\gamma_{s}(t-\tau)} \cos \left[\omega_{s}(t-\tau)\right] Q[\alpha(\tau)] \\
& \bar{B}_{s}=\int_{t_{0}}^{t} d \tau e^{-\gamma_{s}(t-\tau)} \sin \left[\omega_{s}(t-\tau)\right] Q[\alpha(\tau)]
\end{aligned}
$$

The time derivative of Eq. (B8) reads

$$
\begin{aligned}
& \partial_{t} B_{s}=-\gamma_{s} B_{s}-\omega_{s} \bar{B}_{s}+Q[\alpha(t)] \\
& \partial_{t} \bar{B}_{s}=-\gamma_{s} \bar{B}_{s}+\omega_{s} B_{s}
\end{aligned}
$$

Thus the memory functionals of Eq. (B7) satisfy

$$
\partial_{t} \mathcal{B}_{s}=-\gamma_{s} \mathcal{B}_{s}-\frac{i}{\hbar}\left\{c_{s} Q[\alpha(t)]-c_{s}^{*} Q\left[\alpha^{\prime}(t)\right]\right\},
$$

for $s=0,1, \cdots, N_{r}$; while

$$
\begin{aligned}
& \partial_{t} \mathcal{B}_{s}=-\gamma_{s} \mathcal{B}_{s}+\frac{a_{s}}{b_{s}} \omega_{s} \overline{\mathcal{B}}_{s}-\frac{i}{\hbar} a_{s} \mathcal{A}[\boldsymbol{\alpha}(t)] \\
& \partial_{t} \overline{\mathcal{B}}_{s}=-\gamma_{s} \overline{\mathcal{B}}_{s}-\frac{b_{s}}{a_{s}} \omega_{s} \mathcal{B}_{s}
\end{aligned}
$$

for $s=N_{r}+1, \cdots, N_{p}$.

The above equations of motion for $\left\{\mathcal{B}_{s}, \overline{\mathcal{B}}_{s}\right\}$ are closed, with the inhomogeneous terms depending only on the local time. This is right the property for these $\left\{\mathcal{B}_{s}, \overline{\mathcal{B}}_{s}\right\}$ defined in Eq. (B7) to be the IGFs for the desired hierarchy construction. The auxiliary influence functionals $\mathcal{F}_{\mathrm{n}}$, with the labeling index $\mathrm{n}$ of Eq. (16), are now obtained via the IGFs as 9]

$$
\mathcal{F}_{\mathrm{n}}=\frac{1}{\sqrt{\sigma_{\mathrm{n}}}}\left[\prod_{s=0}^{N_{r}} \mathcal{B}_{s}^{n_{s}} \cdot \prod_{s=N_{r}+1}^{N_{p}}\left(\mathcal{B}_{s}^{n_{s}} \overline{\mathcal{B}}_{s}^{\bar{n}_{s}}\right)\right] \mathcal{F}
$$

which specifies the ADO as $\rho_{\mathrm{n}}(t) \equiv \mathcal{U}_{\mathrm{n}}\left(t, t_{0}\right) \rho\left(t_{0}\right)$ with

$$
\mathcal{U}_{\mathrm{n}}\left(\boldsymbol{\alpha}, t ; \boldsymbol{\alpha}_{0}, t_{0}\right) \equiv \int_{\boldsymbol{\alpha}_{0}\left[t_{0}\right]}^{\boldsymbol{\alpha}[t]} \mathcal{D} \boldsymbol{\alpha} e^{i S[\alpha] / \hbar} \mathcal{F}_{\mathrm{n}}[\boldsymbol{\alpha}] e^{-i S\left[\alpha^{\prime}\right] / \hbar}
$$

Included in Eq. (B11) is also a proper scaling parameter $\sigma_{\mathrm{n}}$. This is for the purpose of applying simple and efficient filtering algorithm 13] in HEOM propagation. This parameter scales the specified $\rho_{\mathrm{n}}$ to be of not just the same unit, but also about the same error tolerance as the reduced system density operator $\rho \equiv \rho_{0}$ of primary interest. It is given by [13, 14]:

$$
\sigma_{\mathrm{n}}=\prod_{s=0}^{N_{r}}\left(\left|c_{s}\right|^{n_{s}} n_{s} !\right) \cdot \prod_{s=N_{r}+1}^{N_{p}}\left(\left|a_{s}\right|^{n_{s}} n_{s} !\left|b_{s}\right|^{\bar{n}_{s}} \bar{n}_{s} !\right)
$$

The HEOM [Eqs. (15)-(20)] can now be obtained readily by taking the time derivative on $\rho_{\mathrm{n}}(t)$ or its propagator $\mathcal{U}_{\mathrm{n}}$ of Eq. (B12). Various terms in Eqs. (15) and (17)(20) result from the derivatives on various components in Eqs. (B11) and Eq. (B12), as detailed as follows. The coherent Liouvillian dynamics part in Eq. (15) arises from the derivative of the action functionals in Eq. (B12); The tier-up $\rho_{\mathrm{n}}^{\{+\}}$term [Eq. (20)] is from $\partial_{t} \mathcal{F}=-\frac{i}{\hbar} \mathcal{A}(\mathcal{B \mathcal { F }})$, following by using Eqs. (B6) and (B11), and the operatorlevel form of $\mathcal{A} \cdot=[Q, \cdot]$; Finally, $\Gamma_{\mathrm{n}}, \rho_{\mathrm{n}}^{\{\leftrightarrow\}}$, and $\rho_{\mathrm{n}}^{\{-\}}$ [Eqs. (17)-(19)] collect the decay, swap, and inhomogeneous terms of Eqs. (B10), respectively. Note that the scaling parameters, $\sigma_{\mathrm{n}}$ of Eq. (B13), are also involved. 
tific, Singapore, 2008, 3rd ed.

[3] H. Kleinert, Path Integrals in Quantum Mechanics, Statistics, Polymer Physics, and Financial Markets, World Scientific, Singapore, 2009, 5th ed.

[4] Y. Tanimura, R. Kubo, J. Phys. Soc. Jpn. 58 (1989) 101.

[5] Y. Tanimura, P. G. Wolynes, Phys. Rev. A 43 (1991) 4131.

[6] Y. Tanimura, J. Phys. Soc. Jpn. 75 (2006) 082001.

[7] A. Ishizaki, Y. Tanimura, J. Phys. Soc. Jpn. 74 (2005) 3131.

[8] R. X. Xu, P. Cui, X. Q. Li, Y. Mo, Y. J. Yan, J. Chem. Phys. 122 (2005) 041103.

[9] R. X. Xu, Y. J. Yan, Phys. Rev. E 75 (2007) 031107.

[10] J. S. Jin, X. Zheng, Y. J. Yan, J. Chem. Phys. 128 (2008) 234703.

[11] X. Zheng, J. S. Jin, Y. J. Yan, J. Chem. Phys. 129 (2008) 184112

[12] X. Zheng, J. S. Jin, S. Welack, M. Luo, Y. J. Yan, J. Chem. Phys. 130 (2009) 164708.

[13] Q. Shi, L. P. Chen, G. J. Nan, R. X. Xu, Y. J. Yan, J.
Chem. Phys. 130 (2009) 084105.

[14] Q. Shi, L. P. Chen, G. J. Nan, R. X. Xu, Y. J. Yan, J. Chem. Phys. 130 (2009) 164518.

[15] L. P. Chen, R. H. Zheng, Q. Shi, Y. J. Yan, J. Chem. Phys. 131 (2009) 094502.

[16] J. Xu, R. X. Xu, Y. J. Yan, New J. Phys. In press.

[17] R. X. Xu, B. L. Tian, J. Xu, Q. Shi, Y. J. Yan, J. Chem. Phys. Submitted.

[18] F. Gagel, J. Comput. Phys. 139 (1998) 399.

[19] S. Goedecker, Rev. Mod. Phys. 71 (1999) 1085.

[20] S. Goedecker, Phys. Rev. B 48 (1993) 17573.

[21] D. M. C. Nicholson, G. M. Stocks, Y. Wang, W. A. Shelton, Z. Szotek, W. M. Temmerman, Phys. Rev. B 50 (1994) 14686

[22] D. M. C. Nicholson, X.-G. Zhang, Phys. Rev. B 56 (1997) 12805.

[23] T. Ozaki, Phys. Rev. B 75 (2007) 035123.

[24] A. Croy, U. Saalmann, Phys. Rev. B 80 (2009) 073102. 